\title{
Performance of lung ultrasonography for the diagnosis of community- acquired pneumonia in hospitalized children
}

\author{
Sorin Claudiu Man' ${ }^{1}$, Otilia Fufezan ${ }^{2}$, Valentina Sas ${ }^{1}$, Cristina Schnell ${ }^{1}$
}

${ }^{1} 3^{\text {rd }}$ Pediatric Department, "Iuliu Hațieganu” University of Medicine and Pharmacy, ${ }^{2}$ Department of Radiology, Emergency Clinical Hospital for Children, Cluj-Napoca, Romania

\begin{abstract}
Aims: The objective of the study was to assess the performance of lung ultrasonography (LUS) as compared to chest radiography and the clinical criteria for the diagnosis of pneumonia in children. Materials and methods: This was a retrospective study in which data were collected from medical files of 81 children admitted with a clinical suspicion of pneumonia in which both an LUS and a chest radiograph during the hospitalization were performed. Reference standard used for the diagnosis of pneumonia were chest radiographs (consolidation, parenchymal infiltrates, and interstitial infiltrate) and clinical criteria. LUS findings were reported as normal, parenchymal consolidations and pleural effusions. Results: Radiological pneumonia was reported in 72 of the 81 patients $(88.9 \%)$. LUS identified parenchymal consolidations in 62 cases. LUS correctly identified radiological pneumonia with a sensitivity of $79.2 \%$ and a specificity of $44.4 \%$. The positive predictive value (PPV) was $91.9 \%$ and the negative predictive value (NPV) was $21.0 \%$. When clinical criteria were used as reference standard, the sensitivity, specificity, PPV and NPV of correctly identifying clinical pneumonia cases by LUS (only consolidations) were $80.0 \%, 66.7 \%$, $96.8 \%$ and $21.0 \%$. When indicative for the presence of pneumonia either the ultrasound consolidation or the ultrasound detected pleural effusion were considered when the sensitivity, PPV and NPV increased to 96.0\% (95\%CI: 88.8-99.2), 97.3\% (95\%CI: 92.0-99.1), and 57.1\% (95\%CI: 27.7-82.2), respectively. Conclusions: In our opinion, our findings together with previous ones available in the literature recommend LUS as a valuable investigation for the diagnosis of community-acquired pneumonia in children.
\end{abstract}

Keywords: ultrasonography; chest radiograph; pneumonia; child

\section{Introduction}

Despite available effective interventions to prevent the disease and reduce the associated deaths, pneumonia remains a major health problem in children $[1,2]$. According to data reported by the World Health Organization (WHO), pneumonia represents the leading cause of death in children under the age of 5 years, accounting for $19 \%$ to $29 \%$ of deaths in this age group [2] and 1.6 million deaths per year [3]. The main etiological pathogens reported in epidemiological studies worldwide are

Received 01.03.2017 Accepted 17.04.2017

Med Ultrason

2017, Vol. 19, No 3, 276-281

Corresponding author: Sorin Claudiu Man

3rd Pediatric Department

2-4 Câmpeni Street,

400217 Cluj-Napoca, Romania

Email: claudiu.man@umfcluj.ro
Streptococcus pneumoniae and Haemophilus influenzae type $\mathrm{b}[2,4]$.

Diagnosis of pneumonia remains itself a challenge due to the lack of a universal guideline for its diagnosis. Although chest radiographs are the golden standard for its diagnosis, the radiographs are recommended only for severe and complicated cases to document the presence of parenchymal infiltrates and complications [5-7]. The diagnosis should be based on clinical signs and symptoms of respiratory distress and fever; however, these symptoms have low specificity and may vary according to age [8-10]. Acute phase reactants and microbiological testing are also reserved for complicated and severe pneumonia cases [8-10].

Chest radiographs are frequently used for the classification of pneumonia but the variability in their interpretation in the context of diagnosis of pneumonia in children remains a problem. To address this, WHO issued 
definitions for the diagnosis of radiological pneumonia aiming to increase the accuracy of pneumonia assessment [2]. However, data from a recent epidemiological study from Northern England showed that despite the implementation of the WHO criteria there was still an important $(22 \%)$ inter-observer disagreement in the interpretation of chest radiographs, and this was higher in cases of children below 5 years of age [11]. It has been shown that non-severe pneumonia cases may display or be interpreted as normal chest radiographs or that patchy and perihilar changes as well as pleural effusion may go undetected [11,12]. Thus, human error remains a challenge when interpreting chest radiographs in children and a cause of false negative results and radiological pneumonia underdiagnosis [13].

Due to the limitations described above and the potential adverse effect of ionizing radiation later in life, physicians have turned their attention to lung ultrasonography (LUS) as a safe and accessible option for the diagnosis of pneumonia. Although still limited as the number of studies and number of children enrolled remains small, available prospective clinical studies assessing its diagnostic accuracy when compared to chest radiographs or clinical criteria for the diagnosis of pneumonia in children have shown good results, with a reported sensitivity ranging between $85.7 \%$ and $100 \%$ and specificity between $88.5 \%$ and $100 \%$ [14-16]. Nevertheless, controversies remain with regard to its performance in discriminating the lung consolidations from atelectasis, especially focal atelectasis, and B-lines identification, areas of white lung considered an indicator of perilesional inflammatory edema, which are frequently seen also in patients without pneumonia [17].

The objective of our study was to assess the performance of LUS as compared to chest radiograph and clinical criteria for the diagnosis of pneumonia in children.

\section{Material and methods}

This was a retrospective study in which data from medical files of all children admitted with a clinical suspicion of pneumonia between 2009 and 2014 in the $3^{\text {rd }}$ Pediatrics Clinic, Clinical Emergency Hospital for Children, Cluj-Napoca, Romania were collected. Clinical suspicion of pneumonia was established by the physician based on the institution's protocol, using clinical findings, such as the presence of fever, cough, chest pain, respiratory distress, increased respiratory rate, and breath sounds modification. Additional inclusion criterion was the performance of both chest radiography and of LUS examination in the same patient with a clinical suspicion of pneumonia, irrespective of their findings. Accord- ing to the institution's protocol in cases admitted with a clinical suspicion of pneumonia, a chest radiograph is a mandatory investigation only in younger ages. LUS examination is not a mandatory investigation and may be performed if the treating physician considers that it may provide additional information for guiding pneumonia diagnosis and/or therapy.

Description of chest radiograph and LUS findings was collected from patient files. According to the institution's procedure, chest radiographs were performed and interpreted by an experienced senior radiologist using a commercially available machine (Hofmann Combigraph Quadro, Hofmann X-ray Systems, Germany). In children $<5$ years of age were performed antero-posterior chest radiographs in sitting position. In children $\geq 5$ years of age were performed postero-anterior chest radiographs in standing position. The following findings were considered characteristic for the diagnosis of radiological pneumonia: consolidation, parenchymal or interstitial infiltrate (linear and patchy densities).

LUS was performed before or after the chest radiograph by a senior radiologist experienced in performing ultrasonography, using convex (7-11 MHz) and linear probes (3.5-5 MHz) and Accuvix V20 Medison ultrasound system until 2014 and Toshiba Xario 200 ultrasound system in 2014. Ultrasonographic images were obtained with patients first in a supine and then in a sitting position. Anterior and posterior intercostal spaces were examined in longitudinal and diagonal sections. Findings were reported as normal (defined as normal lung with or without A lines), parenchymal consolidations (defined as subpleural tissue-like or echo-poor region, with or without air-bronchogram) and pleural effusions (defined as hypoechoic or anechoic fluid in the pleural space) [18]. Parenchymal consolidations with or without sonographic air bronchograms identified on LUS were considered suggestive for the presence of pneumonia; additionally, the presence of pleural effusion was also noted.

Results of laboratory investigations (leukocyte count, erythrocyte sedimentation rate [ESR], C-reactive protein [CRP]) and clinical findings (presence of fever, cough, chest pain, respiratory distress, other symptoms, respiratory rate, oxygen saturation on room air, breath sounds) were also collected and included in the analysis.

The gold standard for the diagnosis of pneumonia, i.e. chest computed tomography, is not used in our department as standard of care in children due to ethical considerations related to the radiation dose. Therefore, in our analysis the reference standards used were radiological pneumonia according to the criteria described above and clinical pneumonia. Diagnosis of clinical pneumonia was performed according to the institution's protocol and 
was based on 1) medical history and clinical examination showing the presence of fever, cough, chest pain, respiratory distress, increased respiratory rate, and decreased or absent breath sound, crackles; 2) laboratory investigations - leukocytosis with neutrophilia, increased ESR, increased levels of CRP; and/or 3) pleural effusion as a complication of pneumonia.

The study was conducted according to the Ethical Principles for Medical Research Involving Human Participants as presented in the World Medical Association Declaration of Helsinki. Local Ethics Committee approved the study protocol. Due to the retrospective nature of the study, no informed consent for the collection and the analysis of the data was required and obtained from parents or legal guardians. As per local procedures, at the admittance, parents were asked to sign an informed consent related to the usual care in the institution.

\section{Statistical analysis}

Data was summarized using descriptive statistics: proportions for qualitative variables, mean and standard deviation for continuous variables. The accuracy of LUS to correctly identify the presence of pneumonia as compared to the chest radiograph and clinical diagnosis was assessed calculating sensitivity, specificity, the positive predictive value (PPV), and the negative predictive value (NPV). A p value of less than 0.05 was considered statistically significant. A two-sided 0.05 significance level was applied to all tests. Statistical analysis was carried out using Stata 14 (StataCorp LP, College Station, Texas, US) and Medcalc v. 17.2 (MedCalc Software bvba, Ostend, Belgium).

\section{Results}

Between 2009 and 201497 patients with a clinical suspicion of pneumonia and for whom a LUS was performed were admitted to the $3^{\text {rd }}$ Pediatrics Clinic, Clinical Emergency Hospital for Children, Cluj-Napoca, Romania. No chest radiograph was performed in 16 of these children and they were excluded from the analysis. Thus, we collected data from 81 children (mean age $6.5 \pm 4.7$ years, male $51.5 \%$ ) admitted with a clinical suspicion of pneumonia and for whom both a LUS and a chest radiograph during the hospitalization was performed. Demographic data, clinical presentation and laboratory findings are presented in Table I.

Consolidation, parenchymal infiltrates, and interstitial infiltrate on the chest radiograph was reported in 72 of the 81 patients admitted with clinical suspicion of pneumonia and these cases were considered as having radiological pneumonia (prevalence $88.9 \%$ ). Ultrasound identified peripheral parenchymal consolidations in 62
Table I. The demographic and clinical characteristics of the study group (81 patients).

\begin{tabular}{|c|c|}
\hline Parameter & $\begin{array}{l}\text { Number of } \\
\text { patients }\end{array}$ \\
\hline Male gender & $42(51.9)$ \\
\hline Age (years) & $6.5 \pm 4.7$ \\
\hline Cough & $75(92.6)$ \\
\hline Fever & $68(84.0)$ \\
\hline Respiratory distress & $22(27.2)$ \\
\hline Thoracic pain & $15(18.5)$ \\
\hline $\begin{array}{l}\text { Other } \\
\text { Headache } \\
\text { Wheezing } \\
\text { Abdominal pain } \\
\text { Labial herpes } \\
\text { Congestive heart failure } \\
\text { Vomiting } \\
\text { Muscle pain } \\
\text { Sweating } \\
\text { Asthma }\end{array}$ & $\begin{array}{l}6(7.4) \\
5(6.2) \\
4(4.9) \\
4(4.9) \\
2(2.5) \\
2(2.5) \\
1(1.2) \\
1(1.2) \\
1(1.2)\end{array}$ \\
\hline Respiratory rate & $30.8 \pm 12.2$ \\
\hline Oxygen saturation (\%) & $94.1 \pm 2.7$ \\
\hline $\begin{array}{l}\text { Laboratory investigations } \\
\text { Erythrocytes sedimentation rate }(\mathrm{mm} / \mathrm{h}) \\
\text { Leukocytes counting }(\mathrm{no} / \mathrm{ml}) \\
\text { Neutrophils counting }(\mathrm{no} / \mathrm{ml}) \\
\text { C-reactive protein }(\mathrm{mg} / \mathrm{dl})\end{array}$ & $\begin{array}{l}43.2 \pm 23.4 \\
15238.8 \pm 8576.2 \\
9927.0 \pm 7327.0 \\
9.9 \pm 7.3\end{array}$ \\
\hline Crackles & $57(70.4)$ \\
\hline $\begin{array}{l}\text { Breath sound } \\
\text { Decreased } \\
\text { Absent }\end{array}$ & $\begin{array}{l}44(54.3) \\
3(3.7)\end{array}$ \\
\hline $\begin{array}{l}\text { Chest radiograph findings } \\
\text { Consolidation, parenchymal infiltrates and } \\
\text { interstitial infiltrate } \\
\text { Pleural effusion } \\
\text { Either alveolar infiltrate or pleural ef- } \\
\text { fusion }\end{array}$ & $\begin{array}{l}72(88.9) \\
29(35.8) \\
78(96.3)\end{array}$ \\
\hline Clinical pneumonia & $75(92.6)$ \\
\hline
\end{tabular}

Data are expressed in number $(\%)$ or mean \pm SD. $\mathrm{SD}=$ standard deviation

cases. An example of comparative findings on the chest radiograph and LUS is provided in figure 1. Of these, 57 also had suggestive changes on the chest radiograph (true positive cases), and for 5 the chest radiograph was negative. Of those with a positive chest radiograph, ultrasound did not identify any parenchymal consolidations in 15 cases (Table II). When the reference standard used for the identification of pneumonia was the chest radiograph, LUS correctly identified radiological pneumonia, with a sensitivity of $79.2 \%$ and a specificity of $44.4 \%$. The PPV was $91.9 \%$ and the NPV was $21.0 \%$ (Table III). Pleural effusion was identified on chest radiograph in 29 cases. Of these, LUS identified pleural effusion in 23 cases. 10 additional cases with no pleural collection on chest radiograph were identified by LUS. 
Clinical pneumonia was diagnosed in 75 of the 81 patients (prevalence 92.6\%). LUS identified parenchymal consolidations in 60 of the 75 patients with clinical pneumonia (true positive cases) and 2 additional cases with parenchymal consolidations which were not considered as having clinical pneumonia (false positive cases). In 15 cases, no ultrasound consolidation was identified although they were considered as having clinical pneumonia (false negative cases) and 4 cases with no clinical pneumonia were correctly identified (Table II). When clinical criteria were used as reference standard, the sensitivity, specificity, PPV and NPV of correctly identifying clinical pneumonia casesby LUS were $80.0 \%, 66.7 \%$,

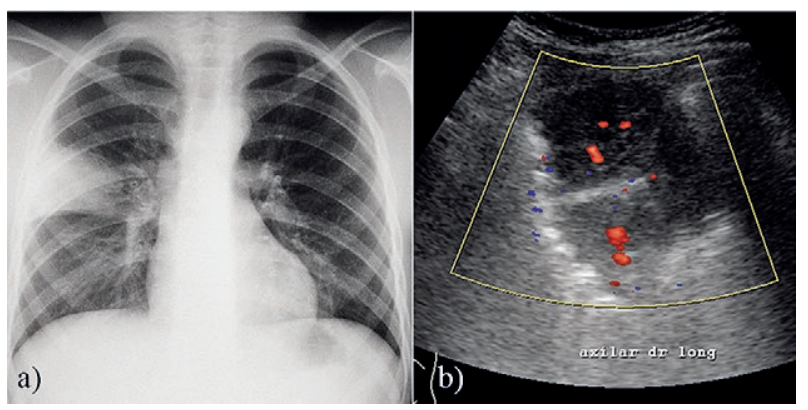

Fig 1. A segmental upper lobe consolidation: a) Chest X ray reveals an opacity in the right upper lobe (well delimited by horizontal fissure); b) Ultrasound performed at the level of the axilla shows a "tissular" pattern of the consolidated segment with vessels inside. In depth there is an irregular border (shred sign), the transition between consolidation and the normal aerated lung

Table II. Agreement between chest radiograph, clinical diagnosis of pneumonia and lung ultrasonography in the study population with suspected community-acquired pneumonia.

\begin{tabular}{lllll}
\hline & $\begin{array}{l}\text { Chest } \mathbf{R x}+ \\
\mathbf{N = 7 2}\end{array}$ & $\begin{array}{l}\text { Chest } \mathbf{R x}- \\
\mathbf{N = 9}\end{array}$ & $\begin{array}{l}\mathbf{C P}+ \\
\mathbf{N = 7 5}\end{array}$ & $\begin{array}{l}\mathbf{C P}- \\
\mathbf{N = 6}\end{array}$ \\
\hline LUS + & 57 & 5 & 60 & 2 \\
LUS - & 15 & 4 & 15 & 4 \\
LUSE+ & - & - & 72 & 2 \\
LUSE- & - & - & 3 & 4 \\
\hline
\end{tabular}

$\mathrm{Rx}=$ Radiograph $; \mathrm{CP}=$ Clinical pneumonia LUS = lung ultrasound, only parenchymal consolidations; LUSE = lung ultrasound, parenchymal consolidations, and pleural effusion; + = present; - = absent; $\mathrm{N}=$ number of patients
$96.8 \%$ and $21.0 \%$, respectively (Table III). When indicative for the presence of pneumonia either the ultrasound consolidation or the ultrasound detected pleural effusionwere considered and the true positive cases identified increased to 72 and false negative cases decreased to 3 . The sensitivity, PPV, and NPV also increased to $96.0 \%$ (95\%CI: 88.8-99.2), 97.3\% (95\%CI: 92.0-99.1), and $57.1 \%$ (95\%CI: 27.7-82.2), respectively. The likelihood ratio for positive ultrasound results was 2.9 (95\% CI: 0.98.9) and the likelihood ratio for a negative ultrasound result was 0.06 (95\%CI: 0.02-0.2).

\section{Discussions}

This study confirms that LUS is a sensitive diagnosis tool in children with suspected community-acquired pneumonia. The results of LUS and clinical pneumonia demonstrated a good agreement. The sensitivity, specificity, and PPV of LUS for the identification of clinical pneumonia when only consolidations detected on LUS were used as a sign of pneumonia were acceptable. The use of both pleural effusion and consolidations for the diagnosis improved the sensitivity, PPV, and NPV for the detection of cases of clinical pneumonia - sensitivity increased from $80.0 \%$ to $96.0 \%$, PPV from $96.8 \%$ to $97.3 \%$, and NPV from $21.0 \%$ to $57.1 \%$. The specificity remained unchanged. In other words, the identification on LUS of either pleural effusion or consolidations correctly identified $96 \%$ of the children with clinical pneumonia, and the likelihood of a patient with a positive finding on LUS to have clinical pneumonia was $97.3 \%$.

Although limited in number and enrolling a small number of children, previously published reports in the literature both in children and adults have shown very good accuracy of LUS for the diagnosis of pneumonia. In a prospective study published by Urbanowska et al [19], LUS had a sensitivity of $93.4 \%$ and PPV of $95.3 \%$ in identification of lung involvement. The study enrolled 106 consecutive children between 1 and 213 months of age, with a suspicion of community-acquired pneumonia, and the diagnosis was performed based on the presence of clinical criteria and at least one radiographic criterion (consolidation, parenchymal infiltrates, linear and patchy

Table III. The ability of lung ultrasonography (consolidation identified) to predict the presence of radiological and clinical pneumonia.

\begin{tabular}{lllllll}
\hline & Se \% (95\%CI) & Sp \% (95\%CI) & PPV\% (95\%CI) & NPV \% (95\%CI) & PLR (95\%CI) & NLR (95\%CI) \\
\hline $\mathrm{RxP}^{*}$ & $79.2(68.0-87.8)$ & $44.4(13.7-78.8)$ & $91.9(86.3-95.4)$ & $21.0(10.1-38.6)$ & $1.4(0.8-2.6)$ & $0.5(0.2-1.1)$ \\
$\mathrm{CP}^{*}$ & $80.0(69.2-88.4)$ & $66.7(22.3-95.7)$ & $96.8(90.6-98.9)$ & $21.0(11.4-35.5)$ & $2.4(0.8-7.5)$ & $0.3(0.1-0.6)$ \\
$\mathrm{CP} \#$ & $96.0(88.8-99.2)$ & $66.7(22.3-95.7)$ & $97.3(92.0-99.1)$ & $57.1(27.7-82.2)$ & $2.9(0.9-8.9)$ & $0.06(0.02-0.2)$ \\
\hline
\end{tabular}

$\mathrm{RxP}=$ Radiological pneumonia; $\mathrm{CP}=$ Clinical pneumonia; *Only ultrasonographic identified consolidation was used in the model. \#Ultrasonography identified consolidation or pleural effusion were used in the model; CI = confidence interval; Se- Sensitivity; Sp- Specificity; PPV = positive predictive value; NPV = negative predictive value; PLR- Positive Likelihood Ratio; NLR- Negative Likelihood Ratio 
densities, or pleural effusion). A meta-analysis including 8 prospective studies performed in neonates and pediatric population and enrolling 765 children showed that LUS had an overall sensitivity of $95 \%$ (95\%CI: 94-97), positive likelihood ratio of 15.3 and negative likelihood ratio of 0.06 for the presence of pneumonia as diagnosed based on clinical criteria and radiographic findings [8].

As compared to other reports, our results showed a lower specificity (66.7\%) and NPV (57.1\%). LUS correctly identified $66.7 \%$ of the patients without clinical pneumonia and $57.1 \%$ of those with a negative finding on LUS did not have clinical pneumonia. However, LUS is not proposed as a single diagnostic tool in children with suspected pneumonia. Its purpose is to support the diagnosis and the ultrasonographical findings, positive or negative, should be interpreted in the context of clinical findings. Previous individual prospective studies enrolling children with suspicion of pneumonia and in which the diagnosis of pneumonia was established using clinical criteria alone or clinical criteria in combination with radiographic findings have reported higher values of specificity, ranging between $96 \%$ and $100 \%$ and NPV of $95.7 \%[19,20]$. The above mentioned meta-analysis which included all available prospective studies in which the confirmation of pneumonia was performed by a combination of clinical findings and chest radiographs or chest CT scan, showed a specificity of LUS of $93 \%$ (95\%CI: 89.6-95.6) [8].

Previous studies performed both in children and adults have shown that LUS is not inferior to chest radiographs in identifying lung abnormalities in patients with a clinical suspicion of pneumonia [15,16,18,19,21-24]. Urbanowska et al [19] showed a high agreement between LUS and chest radiographs, with a Cohen kappa coefficient of agreement of 0.89. Esposito et al [15] also found a high accuracy of LUS as compared to chest radiographs, with a sensitivity of $97.9 \%$ and a specificity of $94.5 \%$. When radiological findings were used as a test reference, Shah et al [16] showed sensitivity and specify of LUS of $86 \%$ and $89 \%$, respectively. In our study, compared to chest radiographs, LUS correctly identified 57 of the children with radiological pneumonia, while in 15 cases no condensation was visible by LUS. Among the reasons for LUS not detecting the lung modifications are the location of lesions in areas not easily reached by ultrasound beam or lesions of small dimensions that do not extend to pleura [24]. In our study the performance of LUS when using chest radiographs as a reference standard was $79.2 \%$ (sensitivity) and $44.4 \%$ (specificity), respectively. These results show a partial agreement between LUS and chest radiographs. It should also be noted that LUS identified 5 additional cases in which no consolidation was visible on chest radiographs. Similar findings on higher performance of LUS as compared to chest radiographs in certain cases have been reported in the literature and among explanations is the reduced dimension of the lung consolidations, too small to be identified by radiography, and the location of the lesions behind the heart or mediastinum [16,24]. Head-to-head comparisons of chest radiological and LUS findings, with chest computed tomography (CT) as a reference standard, are available only in adults and showed a higher accuracy of LUS in the detection of lung changes [25]. In a clinical study performed in 179 adults with suspected community-acquired pneumonia, and in which CT was used as reference standard, LUS performed better as compared to the chest radiograph in identifying CT changes characteristic for pneumonia, with a sensitivity of $94.6 \%$ vs. $77.7 \%$, a specificity of $98.5 \%$ vs. $94.0 \%$ and an accuracy of $96.1 \%$ vs. $83.8 \%$ [25]. As in our patients no chest CT was performed for the diagnosis of pneumonia, it remains an open question on whether cases with only LUS findings are indeed false positive cases or true cases undetected by chest radiography. In our study, LUS also performed better than chest radiography for the detection of pleural effusion - 10 additional cases with pleural effusion were identified by LUS. Similar results of a higher performance of LUS in the identification of pleural effusion when compared to chest radiographs have been previously reported, with LUS identifying effusion in $54.3 \%$ of the patients and chest radiographs in only $12.1 \%$ of the patients [19].

Our study has several limitations which may have influenced the results and that should be acknowledged. The study was a retrospective one and no standard protocol for the investigations was in place before the performance of all diagnostic procedures. The radiologist performing the ultrasonography was not blinded to the suspicion of clinical pneumonia or to the chest radiograph results. LUS and chest radiograph were not performed on the same day or in a certain order and this may have been reflected in a lack of concordance between the results due to improvements or worsening of the disease. Another limitation is the use of the chest radiograph as a reference standard for the diagnosis of radiological pneumonia instead of CT. CT may have helped for the clarification of 'false positive' cases with no findings on the chest radiograph and positive on LUS. However, due to the high dose of radiation, CT cannot be used for the detection of pneumonia in clinical studies involving children.

\section{Conclusions}

In our opinion our findings together with previous ones available in the literature recommend LUS as a valuable 
tool for the diagnosis of community-acquired pneumonia in children. Chest radiographs may not be required in certain cases with clinical findings suggestive of communityacquired pneumonia and specific LUS abnormalities.

\section{Conflict of interest: none}

\section{References}

1. Wardlaw T, Salama P, Johansson EW, Mason E. Pneumonia: the leading killer of children. Lancet 2006;368:1048-1050.

2. UNICEF, World Health Organization. The forgotten killer of children, 2006. Available at: http://apps.who.int/iris/ bitstream/10665/43640/1/9280640489 eng.pdf. Last accessed 21 Feb 2017.

3. Black RE, Cousens S, Johnson HL, et al. Global, regional, and national causes of child mortality in 2008: a systematic analysis. Lancet 2010;375:1969-1987.

4. Levine OS, O'Brien KL, Deloria-Knoll M, et al. The Pneumonia Etiology Research for Child Health Project: a 21st century childhood pneumonia etiology study. Clin Infect Dis 2012;54(Suppl 2):S93-S101.

5. Harris M, Clark J, Coote N, et al. British Thoracic Society guidelines for the management of community acquired pneumonia in children: update 2011.Thorax 2011;66(Suppl 2):ii1-ii23.

6. Bradley JS, Byington CL, Shah SS, et al. The management of community-acquired pneumonia in infants and children older than 3 months of age: clinical practice guidelines by the Pediatric Infectious Diseases Society and the Infectious Diseases Society of America. Clin Infect Dis 2011;53:e25-e76.

7. Iorio G, Capasso M, De Luca G, et al. Lung ultrasound in the diagnosis of pneumonia in children: proposal for a new diagnostic algorithm. Peer J 2015;3:e1374.

8. Pereda MA, Chavez MA, Hooper-Miele CC, et al. Lung ultrasound for the diagnosis of pneumonia in children: a meta-analysis. Pediatrics 2015;135:714-722.

9. Ebell MH. Clinical diagnosis of pneumonia in children. Am Fam Physician 2010;82:192-193.

10. Shah S, Sharieff GQ. Pediatric respiratory infections. Emerg Med Clin North Am 2007;25:961-979.

11. Elemraid MA, Muller M, Spencer DA, et al. Accuracy of the interpretation of chest radiographs for the diagnosis of paediatric pneumonia. PLoS One 2014;9:e106051.
12. Hazir T, Nisar YB, Qazi SA, et al. Chest radiography in children aged 2-59 months diagnosed with non-severe pneumonia as defined by World Health Organization: descriptive multicentre study in Pakistan. BMJ 2006;333:629.

13. Berlin L. Accuracy of diagnostic procedures: has it improved over the past five decades? AJR Am J Roentgenol 2007; 188:1173-1178.

14. Copetti R, Cattarossi L. Ultrasound diagnosis of pneumonia in children. Radiol Med 2008;113:190-198.

15. Esposito S, Papa SS, Borzani I, et al. Performance of lung ultrasonography in children with community-acquired pneumonia. Ital J Pediatr 2014;40:37.

16. Shah VP, Tunik MG, Tsung JW. Prospective evaluation of point-of-care ultrasonography for the diagnosis of pneumonia in children and young adults. JAMA Pediatr 2013; $167: 119-125$.

17. Tomà $\mathrm{P}$. Sonographic diagnosis of pneumonia: it is time to change. Br J Radiol 2017;90:20160799.

18. Caiulo VA, Gargani L, Caiulo S, et al. Lung ultrasound characteristics of community-acquired pneumonia in hospitalized children. Pediatr Pulmonol 2013;48:280-287.

19. Urbankowska E, Krenke K, Drobczyński Ł, et al. Lung ultrasound in the diagnosis and monitoring of community acquired pneumonia in children. Respir Med 2015;109:12071212.

20. Reali F, Sferrazza Papa GF, Carlucci P, et al. Can lung ultrasound replace chest radiography for the diagnosis of pneumonia in hospitalized children? Respiration 2014;88:112115.

21. Parlamento S, Copetti R, Di Bartolomeo S. Evaluation of lung ultrasound for the diagnosis of pneumonia in the ED. Am J Emerg Med 2009;27:379-384.

22. Reissig A, Kroegel C. Sonographic diagnosis and follow-up of pneumonia: a prospective study. Respiration 2007;74:537-547.

23. Testa A, Soldati G, Copetti R, Giannuzzi R, Portale G, Gentiloni-Silveri N. Early recognition of the 2009 pandemic influenza A (H1N1) pneumonia by chest ultrasound. Crit Care 2012;16:R30.

24. Ho MC, Ker CR, Hsu JH, Wu JR, Dai ZK, Chen IC. Usefulness of lung ultrasound in the diagnosis of community-acquired pneumonia in children. Pediatr Neonatol 2015;56:40-45.

25. Liu XL, Lian R, Tao YK, Gu CD, Zhang GQ. Lung ultrasonography: an effective way to diagnose community-acquired pneumonia. Emerg Med J 2015;32:433-438. 